5. Lindsay J, Smith MA, Light JA. Torsades de pointes associated with antimicrobial therapy for pneumonia. Chest 1990;98:222-223.

6. Stein KM, Haronian H, Mensah GA, Acosta A, Jacobs J, Kligfield P. Ventricular tachycardia and torsade de pointes complicating pentamidine therapy of Pneumocystis carinii pneumonia in the acquired immunodeficiency syndrome. Am J Cardiol 1990;66:888-889.

7. HC Bazett. An analysis of time relations of electrocardiograms. Heart 1920;7:353-370.

8. Farb A, Devereux RB, Kligfield P. Day-to-day variability of voltage measurements used in electrocardiographic criteria for left ventricular hypertrophy. $J$ Am Coll Cardiol 1990;15:618-623.

9. Merri M, Benhorin J, Alberti M, Locati E, Moss AJ. Electrocardiographic quantitation of ventricular repolarization. Circulation 1989;80:1301-1308.

10. Tamura K, Tamura T, Yoshida S, Inui M, Fukuhara N. Transient recurrent ventricular fibrillation due to hypopotassium with special note on the U wave. $J p n$ Heart $J$ 1967;8:652-660.

11. Isner JM, Sours HE, Paris AL, Ferrans VJ, Roberts WC. Sudden unexpected death in avid dieters using the liquid-protein-modified-fast diet: observations in 17 patients and the role of the prolonged QT interval. Circulation 1979;60: 1401-1412.

12. Topol EJ, Lerman BB. Hypomagnesemic torsades de pointes. Am J Cardiol 1983;52:1367-1368.

13. Roden DM, Woosley RL, Primm RK. Incidence and clinical features of the quinidine-associated long QT syndrome: implications for patient care, Am Heart $J$ 1986;111:1088-1093.

14. Gittleman IW, Thorner MC, Griffith GC. The QT-interval of the electrocardiogram in acute myocarditis in adults with autopsy correlation. Am Heart $J$ 1951:41:78-90.

15. Cohen IS, Anderson DW, Virmani R, Reen BM, Macher AM, Sennesh J, DiLorenzo P, Redfield R. Congestive cardiomyopathy in association with the acquired immunodeficiency syndrome. $N$ Engl J Med 1986;315:628-630.

16. Surawicz B, Braun HA, Crum WB, Kemp RL, Wagner S, Bellett S. Quantitative analysis of the electrocardiographic pattern of hypopotassemia. Circulation 1957;16:750-763.

\title{
Noncapturing Stimuli During the Basic Drive Shorten Ventricular Refractoriness
}

Jonathan J. Langberg, MD, Hugh Calkins, MD, João Sousa, MD, Rafel El-Atassi, MD, and Fred Morady, MD

$\mathrm{S}$ tudies of ventricular programmed stimulation in animals ${ }^{1}$ and a recent study in humans ${ }^{2}$ showed that increasing the stimulation intensity of the basic drive train (S1) shortened the effective refractory period (ERP). This effect was independent of the intensity of the extrastimulus (S2). The mechanism of this shortening of refractoriness by high-current intensity is unknown. The effect disappears when the sites of pacing and cxtrastimulation are scparated by $>2 \mathrm{~cm},{ }^{1}$ suggesting that high-current stimulation shortens refractoriness through changes in the sequence of local activation. Local sympathetic activation may also play a role, because autonomic blockade significantly attenuates the shortening in refractoriness that occurs with an increase in the strength of the current of the basic drive train. ${ }^{2}$ This study better defines the mechanism by which intense stimulation shortens refractoriness. High-current intensity stimuli were applied during the absolute refractory period of the basic drive train during measurement of ERP. This allowed evaluation of the effect of stimulation intensity on ventricular refractoriness independent of changes in local activation sequence.

This study was performed during the course of a clinically indicated electrophysiologic test in $20 \mathrm{pa-}$ tients. Inclusion criteria were as follows: (1) absence of known structural heart disease; (2) discontinuation of antiarrhythmic drugs $\geq 5$ half-lives before the test; (3) absence of inducible, sustained ventricular tachycardia/fibrillation; (4) absence of ventricular ectopy during overdrive pacing; and (5) stable ventricular

From the Department of Internal Medicine, Division of Cardiology, University of Michigan Medical Center, 1500 East Medical Center Drive, B1 F245, Ann Arbor, Michigan 48109-0022. Manuscript received February 21, 1991; revised manuscript received and accepted June 19, 1991. capture threshold $\leq 1 \mathrm{~mA}$. The study included 10 men and 10 women (mean age 38 years, range 15 to 77 ). Documented supraventricular tachycardia was the indication for study in 18 patients. One patient was being tested because of unexplained syncope, and another had been resuscitated from an episode of ventricular fibrillation.

Electrophysiologic testing was performed in the fasting, unsedated state after informed, written consent had been obtained. The study protocol was approved by the Human Research Committee. Two or three 6Fr quadripolar electrode catheters (Bard Electrophysiology) were inserted into a femoral vein and positioned in the heart as clinically indicated. Surface and intracardiac electrograms were recorded on a Siemens-Elema Mingograf 7 recorder.

The experimental protocol was performed at the conclusion of the clinical test. A 6 Fr quadripolar electrode catheter with $1 \mathrm{~cm}$ interelectrode spacing was positioned in the apex of the right ventricle. Bipolar pacing, with the distal electrode serving as the cathode and the adjacent ring electrode (pole 2) as the anode, was used throughout. The output of a stimulator (Bloom Associates, Narberth, Pennsylvania) triggered 2 stimulus isolation units (Bloom Associates) that delivered $2 \mathrm{~ms}$ square wave pulses with output currents continuously variable from 0 to $10 \mathrm{~mA}$. To deliver conditioning stimuli of varying intensity while maintaining the intensity of S1 constant, these 2 stimulus isolation units were connected parallel to the distal poles of the right ventricular electrode catheter (Figure 1). Testing under load with a pacing systerns analyzer showed no change in pulse width or amplitude from either unit as a result of coupling the outputs together. 
Programmed stimulation was performed with a 10 beat basic drive train at a cycle length of 500 or 600 $m s$. The intensity of the currents of $S 1$ and $S 2$ were maintained at twice late diastolic threshold (mean 0.5 $\pm 0.1 \mathrm{~mA}$ ), and a 2 second intertrain interval was used throughout the study. To eliminate confounding effects due to variation in mean cycle length, refractoriness was assessed by introducing $S 2$ at a coupling interval less than ERP and by incrementing the S2 coupling interval in steps of 2 ms until capture. ${ }^{3}$ To ensure steady state conditions, programmed stimulation was performed for 1 minute before each ERP measurement. ERP was defined as the longest S1-S2 interval that was consistently unsuccessful in evoking a ventricular depolarization.

After ERP was measured at baseline, the same programmed stimulation protocol was repeated with $5 \mathrm{~mA}$ conditioning stimuli introduced $100 \mathrm{~ms}$ after each of the first 9 SI s of the basic drive (Figure 1). To prevent direct electrotonic interaction between the conditioning stimulus and $S 2$, the tenth and final beat of the basic drive train had no conditioning stimulus. ${ }^{4}$ ERP was next measured during application of $10 \mathrm{~mA}$ conditioning stimuli. Finally, ERP determination was repeated without conditioning stimuli to assess the stability of refractoriness over time.

Patients 11 through 20 had this protocol performed before and 10 minutes after pharmacologic autonomic blockade. Atropine and propranolol were administered intravenously in doses of 0.04 and 0.2 $\mathrm{mg} / \mathrm{kg}$, respectively. These doses have been shown to block the chronotropic effects of isoproterenol. ${ }^{5}$ Intrinsic heart rate was measured at the beginning and end of this phase of the study to assess the stability of the autonomic blockade.

Results are expressed as mean \pm standard deviation. Comparison of ventricular ERPs with and without conditioning stimuli was performed using analysis of variance for repeated measures. Comparison of intrinsic heart rates at the beginning and end of the pacing protocol during autonomic blockade was performed using Student's $\mathrm{t}$ test for paired variables. A p value $<0.05$ was considered significant.

ERP decreased from $241 \pm 12$ at baseline to $236 \pm$ $13 \mathrm{~ms}$ after the addition of $5 \mathrm{~mA}$ conditioning stimuli to the basic drive train $(p<0.05$; Figure 2). Mean
FIGURE 1. Apparatus and pacing protocol. To introduce conditioning stimuli of varying intensities while maintaining $\mathbf{S 1}$ constant at twice threshold, 2 stimulus isolation units were connected in parallel to the distal poles of an electrode catheter in the right ventricular apex. Conditioning stimuli were applied $100 \mathrm{~ms}$ after all but the last beat of the basic drive train. $\mathbf{S}_{\mathbf{c}}=$ conditioning stimulus; $\mathbf{S 1}=$ basic drive train; $\mathbf{5 2}$ = extrastimulus.
FIGURE 2. Effects of high-current strength conditioning stimuli on ventricular effective refractory period (VERP) before and after autonomic blockade. Results are expressed as percentage of baseline VERP. *p $<0.05$ vs baseline; * $p<0.05$ vs VERP measured with $5 \mathrm{~mA}$ conditioning stimuli.
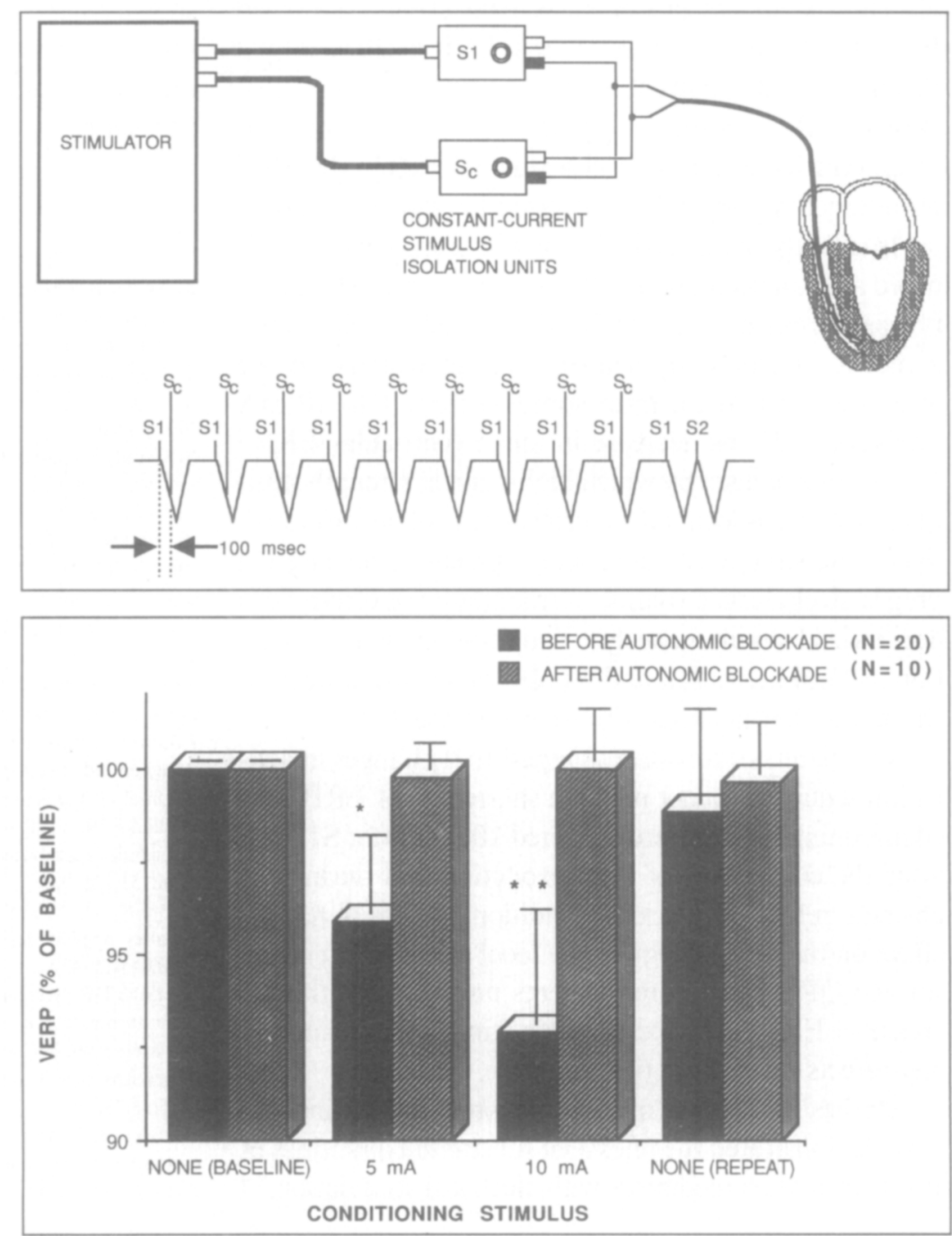
ERP was significantly less $(224 \pm 13 \mathrm{~ms})$ when the measurement was performed with $10 \mathrm{~mA}$ conditioning stimuli $(p<0.05)$. A final ERP measurement without conditioning stimuli was not significantly different from baseline ( $239 \pm 12 \mathrm{~ms})$.

Patients 11 through 20 received a mean of $2.7 \pm$ $0.6 \mathrm{mg}$ of atropine and $14 \pm 3 \mathrm{mg}$ of propranolol. Ten minutes later, at the beginning of the ERP measurements, mean intrinsic heart rate was $99.3 \pm 10$ heats/min. At the end of the pacing protocol, mean heart rate was $99.8 \pm 17$ beats/min ( $p=$ not significant). Baseline ERP during autonomic blockade was $231 \pm 18 \mathrm{~ms}$. There was no significant change in ERP with the addition of 5 or $10 \mathrm{~mA}$ conditioning stimuli $(231 \pm 17$ and $231 \pm 16 \mathrm{~ms}$, respectively). The repeat baseline measurement of ERP without conditioning stimuli was $230 \pm 17$ ( $p=$ not significant versus baseline; Figure 2).

The results of this study demonstrate that high-current intensity stimuli that do not result in ventricular capture can nevertheless shorten ventricular refractoriness. Measurements of ERP were made before and during the addition of conditioning stimuli applied $100 \mathrm{~ms}$ after S1. At an intensity of $5 \mathrm{~mA}$, these stimuli shortened ERP by a mean of $9.8 \pm 5.1 \mathrm{~ms}$. ERP decreased by a mean of $17.1 \pm 7.5 \mathrm{~ms}$ at a conditioning stimulus intensity of $10 \mathrm{~mA}$. This shortening effect of high-current stimuli on ventricular refractoriness was completely abolished by autonomic blockade with atropine and propranolol.

In a study of epicardial pacing in dogs, Avitall et $\mathrm{al}^{1}$ noted a mean decrease in ERP of $23 \mathrm{~ms}$ when S1 intensity was increased from 1 to 10 times threshold. Similarly, a recent clinical study showed that an increase in the intensity of the drive train stimulus from 1 to $10 \mathrm{~mA}$ produced a $22 \mathrm{~ms}$ decrease in mean ventricular ERP. ${ }^{2}$

The mechanism by which high-current strength stimulation shortens local refractoriness has not been defined. When the strength of the pacing current is increased, a progressively larger volume surrounding the electrode is depolarized simultaneously. ${ }^{6}$ The resultant synchronization of local activation and repolarization would tend to shorten action potential duration and the ERP.?

The results of this study suggest that changes in activation sequence do not mediate shortening of ERP. The conditioning stimuli were delivered $100 \mathrm{~ms}$ after S1, long after the completion of local excitation and during the absolute refractory period. Conditioning stimuli had no effect on the morphology of the local electrogram or the surface QRS. Therefore, it seems unlikely that the decrease in ERP produced by these stimuli was related to any effects on local activation.

Studies of isolated myocardial preparations in vitro have demonstrated the release of substantial quantities of autonomic neuromediators with electrical stimulation. ${ }^{8,9}$
In a study of ventricular fibrillation threshold in dogs, high-frequency pacing produced effects that appeared to be mediated by local release of norepinephrine. ${ }^{10}$ The magnitude of autonomic effects was proportional to the intensity of stimulation.

The shortening of ERP by conditioning stimuli was completely reversed by autonomic blockade, which is consistent with the hypothesis that intense stimulation activates sympathetic nerve terminals and causes local release of norepinephrine. An electrical effect on autonomic fibers would be independent of the cardiac cycle and could account for the electrophysiologic effects of stimuli applied during the absolute refractory period.

This study shows that the strength of the current of stimulation has a significant effect on ventricular refractoriness independent of the strength-interval relationship. Therefore, changes in the intensity of programmed stimulation may result in variability in measured ERP due to fluctuation in local catecholamine release. This suggests that use of a constant stimulus intensity may be preferable to the arbitrary choice of twice diastolic threshold and may enhance reproducibility. Increasing the intensity of the current during programmed stimulation has been shown to promote the induction of nonclinical arrhythmias. ${ }^{11,12}$ The results of this study suggest that the local release of catecholamines may contribute to this phenomenon.

1. Avitall B, Levine IIJ, Naimi S, Donahue RP, Pauker SG, Adam D. Local effects of electrical and mechanical stimulation on the recovery properties of the canine ventricle. Am J Cardiol 1982;50:263-270.

2. Langberg JJ, Calkins H, Sousa J, El-Atassi R, Morady F. Efrects of drive train stimulus intensity on ventricular refractoriness in humans (abstr). $P A C E$ $1991 ; 14: 626$.

3. Morady F, Kadish AH, Kushner JA, Toivonen LK, Schmaltz S. Comparison of ventricular refractory periods determined by incremental and decremental scanning of an extrastimulus. PACE 1989;12:546-554.

4. Prystowsky FN, Zipes DP. Inhibition in the human heart. Circulation 1983;68:707-713.

5. Jose AD, Taylor RR. Autonomic blockade by propranolol and atropine to study intrinsic myocardial function in man. $J$ Clin Invest 1989;48:2019-2031.

6. Frazier DW, Krassowska W, Chen P-S, Wolf PD, Dixon EG, Smith WM, Ideker RE. Extracellular field required for excitation in three-dimensional anisotropic canine myocardium. Circ Res 1988;63:147-164.

7. Osaka T, Kodama I, Tsuboi N, Toyama J, Yamada K. Effects of activation sequence and inisotropic cellular geometry on the repolarization phase of action potential of dog ventricular muscles. Circulation 1987;76:226-236.

8. Blinks JR. Field stimulation as a means of effecting the graded release of autonomic transmitters in isolated heart muscle. I Phormarol Fxp Ther 1966;151:221-235.

9. Brady AJ, Abbott BC, Mommaerts WFHM. Inotropic effects of trains of impulses applied during the contraction of cardiac muscle. $J$ Gen Physiol 1960;44:415-432.

10. Euler DE. Norepinephrine release by ventricular stimulation: effect on fibrillation threshold. Am I Physiol 1980;238:H-406 H-413.

11. Morady F, DiCarlo LA, Liem LB, Krol RB, Baerman JM. Effects of high stimulation current on the induction of ventricular tachycardia. Am $J$ Cardiol 1985;56:73-78.

12. Brugada $P$, Abdollah $H$, Wellens HJJ. Sensitivity of a ventricular stimulation protocol using two ventricular extrastimuli at twice diastolic threshold and $20 \mathrm{~mA}$. $J$ Am Cull Cardiol 1984;3:609. 